\title{
Bildung im Alter in sozialen Welten - diesseits und jenseits von Dichotomien
}

\author{
Ines Himmelsbach
}

\begin{abstract}
Zusammenfassung: In der erziehungswissenschaftlichen Diskussion um das Altern haben bislang Prozesse des Stillstands und des Verlusts wenig an Bedeutung erlangt. Im Vordergrund standen vielmehr Aktivierung und an Steigerung orientierte Entwicklung. Die vorgestellte empirische Studie nähert sich aus erziehungswissenschaftlicher Sicht der Frage des individuellen wie institutionellen Umgangs mit Verlusten und Grenzen im Alternsprozess (am Beispiel einer im Alter eintretenden Sehbehinderung, der Makuladegeneration). Die Beschäftigung mit diesem Gegenstand verlangt nach einem theoretischen Rahmen, der sowohl individuelle Lebenswelten in den Blick nimmt und gleichzeitig deren institutionell und sozial strukturierte Infrastrukturen. Dafür arbeitet die qualitativ angelegte Studie mit der Heuristik der „,sozialen Welt“. In diese ist eine Vielzahl pädagogischer Formen eingebettet, auf die von den Befragten jeweils unterschiedlich Bezug genommen wird. Die Relationierung von Akteursperspektiven innerhalb der untersuchten sozialen Welt zeigt dabei auf, dass man den Umgang mit einer altersbedingten Kompetenzeinbuße nicht angemessen begreifen kann, wenn man lineare Vorstellungen pädagogischen Handelns im Sinne einer Steigerung zugrunde legt. Zudem zeigt sich, dass das Pädagogische in sozialen Formen institutionalisiert ist, die eng mit nicht-pädagogischen Handlungsformen verschränkt sind.
\end{abstract}

Schlüsselwörter: Bildung im Alter · Makuladegeneration · Formen des Pädagogischen · Lernen Älterer · Altenbildung · Sehbehinderung · Gerontologie $\cdot$ Soziale Welt

\section{Education in late adulthood embedded in social worlds - Dichotomies and beyond}

Abstract: The debates on ageing in educational sciences have neglected processes of standstill
and loss in the past. The focus was instead on development based on activation and progression.
The study presented here approaches the question of individual and institutional treatment of loss
and constraints during the ageing process from an educational perspective (using the example of
age-related visual impairment). An investigation of this subject requires a theoretical concept,
which considers the individual's life-worlds as well as the institutional and socially structured
infrastructure they are embedded in. For this aim this study, working with a qualitative approach,
uses the heuristic of "social worlds". These worlds encompass a multitude of pedagogic forms,

Online publiziert: 22.12 .2009

(C) Die Autoren 2009. Dieser Artikel ist auf springerlink.com mit Open Access verfügbar.

Dr. I. Himmelsbach $(\bowtie)$

Forum Alterswissenschaften und Alterspolitik

Fachbereich Erziehungswissenschaften, Johann Wolfgang Goethe Universität Frankfurt

Robert-Mayer-Str. 1, 60325 Frankfurt, Deutschland

E-Mail: Himmelsbach@em.uni-frankfurt.de 
to which the respondents relate differently. Considering such relations within the framework of social worlds from an actor-perspective demonstrates that it is not possible to deal with age-related loss of competences by linear conceptions of pedagogic action as progression. Furthermore, it can be shown that the pedagogic actions are institutionalized in social forms, which are closely linked to non-pedagogic forms of action.

Keywords: Age-related macular degeneration · Andragogy · Education in late adulthood Gerontology $\cdot$ Pedagogic forms $\cdot$ Visual impairment $\cdot$ Social worlds

\section{Altern zwischen Kompetenz und Defizit}

Im öffentlichen Diskurs ist das Bild vom Altern häufig geprägt durch stereotype Vorstellungen, die sich polar an Konzepten von Kompetenz und Defizit ausrichten: Ältere sind entweder ,junge‘ agile oder ,alte' gebrechliche Alte. In der gerontologischen Debatte, die vor allem durch die Entwicklungspsychologie der Lebensspanne geprägt ist, wird dahingegen Altern als dynamischer Prozess bestimmt, der sowohl Gewinne als auch Verluste umfasst (vgl. Baltes et al. 2006). Mit grundlegenden Bestimmungen, die Wahl und Heyl (2004) als ,essentials“ der Gerontologie beschreiben, überwindet die Gerontologie eine dichotome Unterscheidung nach Kompetenz und Defizit. Altern ist als Veränderungsgeschehen multidimensional und multidirektional. Das bedeutet, dass Altern auf verschiedenen Ebenen (körperlich, sozial, umweltbezogen) verläuft und jeweils unterschiedliche Entwicklungsverläufe (Veränderung, Stabilität) nehmen kann. Altern ist zudem ein lebenslanger und biografisch verankerter Prozess. Äußere Einflussfaktoren, wie die soziale und die physische Umwelt (in Form von Wohnform oder Infrastruktur), sind zu berücksichtigen (Wahl u. Heyl 2004). Die Ausdifferenzierung des Konzeptes von Altern als komplexes und viel dimensioniertes Geschehen gelang maßgeblich durch (längsschnittliche) empirische Studien der Bonner Längsschnittstudie des Alterns (BOLSA), der Berliner Altersstudie (BASE) oder aber der Seattle Longitudinal Study (SLS). Diese konnten durch die Kombination von psychologischen und medizinischen Fragestellungen nachweisen, dass die auf Mittelwertsvergleichen basierenden Ergebnisse einer biologisch orientierten Defizitthese des unidirektionalen Altersabbaus nicht tragfähig sind, sondern sie müssen durch eine Sicht auf die inter- und intraindividuelle Variabilität des Alterns abgelöst werden (vgl. Lehr et al. 1987; Mayer u. Baltes 1996; Schaie u. Baltes 1996). Gerontologische Ansätze richten somit die Pole von Kompetenz und Defizit differenzierend aus, wobei Ressourcen/Kompetenzen des Alterns insbesondere für das dritte Lebensalter hervorgehoben werden, wohingegen davon ausgegangen wird, dass sich das Gleichgewicht von Gewinnen und Verlusten mit zunehmendem Alter ,zuungunsten der Gewinne" verschiebt (Staudinger 2008). Trotz dieser zeitlichen Dimensionierung wird der Prozess des Alterns als kontinuierliche Interaktion zwischen Biologie, Kultur und Person aufgefasst und ist damit veränderbar (vgl. ebd.). Entwicklungsziele des Alterns lassen sich dabei mit den von Baltes formulierten drei Entwicklungszielen des Wachstums, der Aufrechterhaltung (einschließlich Wiederherstellung) und der Verlustregulation (Baltes 1997) konkretisieren.

Stellt man auf Bildung im Alternsprozess ab, so stößt man auf das Konzept lebenslangen Lernens. Allerdings ist dieses nach wie vor als diffuser Begriff zu betrachten, der 
durch die Gleichzeitigkeit von Instrumentalisierung und Emanzipation gekennzeichnet ist (vgl. Alheit u. Dausien 2002, S. 566). Er beruht somit gleichzeitig auf Aktivierung (als Aufruf zum Lernen) und Freiwilligkeit (als individuelles und selbständiges Lernen). Dies gilt auch für die Phase des Alterns, für welche Lebenslanges Lernen sozialpolitisch als Integrations- und Partizipationsmodus propagiert wird (BMFSFJ 2005). Lernen wird mit der Chance verbunden, auch nach der Verrentung partizipativ (bspw. durch ein ehrenamtliches Engagement) an der Gesellschaft teilzuhaben, und mit der Funktion verknüpft, die eigene Selbständigkeit möglichst lange aufrecht zu erhalten. In dieser Definition setzt Lernen im Alter vor allem auf Wachstum, wird also kompetenzsteigernd verstanden.

Aber nicht allein sozialpolitische Forderungen gehen in diese Richtung. Auch die erziehungswissenschaftliche Diskussion zum Altern hat bislang kaum Elemente des Stillstands und des Verlustes betont. Erst in jüngeren Thesen wird die Überbetonung der „Aktivierung“ (Kolland 2008, S. 175) hinterfragt (vgl. dazu auch Breinbauer 2007; Karl 2008). Noch offen und empirisch kaum beantwortet, ist die Frage des Umgangs mit Verlusten und Grenzen des Alternsprozesses aus pädagogischer Sicht. So fordert etwa Kolland (2008), der einseitigen Aktivierung entgegnend, die Altenbildung auf, „Irritationen im Alltäglichen als Gelegenheit für Lernprozesse wahrzunehmen“ (S. 176). Fragt man im Anschluss an dieses Postulat nach der Gestalt von Lernprozessen im Alltag, verlangt dies einen theoretischen Rahmen, der sowohl individuelle Lebenswelten in den Blick bekommt als auch institutionelle und soziale Welten, die den Umgang mit den Erfahrungen des Alterns (mit-)bedingen.

Ein Versuch zu einer derartigen empirischen Bestimmung des Lernens Älterer wurde in einer Studie zum individuell-institutionellen Umgang mit eingeschränkter Handlungsfähigkeit als einem Kennzeichen des Alterns unternommen (vgl. Himmelsbach 2009), deren Ergebnisse in diesem Beitrag aus einer erziehungswissenschaftlichen Perspektive erörtert werden. ${ }^{1}$ Zunächst werden der theoretische Rahmen, die Fragestellung und methodischen Grundlagen der Studie vorgestellt, mit der Erläuterung des Konzepts der sozialen Welt im Mittelpunkt (2). Deren Technologie wird im folgenden Abschnitt auf drei zunehmend abstrakter werdenden Ebenen analysiert (3). Abschließend werden die theoretischen Perspektiven skizziert, die sich aus diesen Analysen für den erziehungswissenschaftlichen (und gerontologischen) Diskurs zum Altern eröffnen.

\section{Eine empirische Studie über das Altern am Beispiel eingeschränkter Handlungsfähigkeit}

Für eine erziehungswissenschaftliche Betrachtung des Alterns wurde in der vorgestellten Studie ein Feld ausgewählt, das die Ambivalenz des Alterns zwischen Wachstum und Verlustregulation beispielhaft repräsentieren kann: Der Umgang mit einer alterspezifischen chronischen Erkrankung (altersbedingte Makuladegeneration, AMD). An dieser Erkrankung kann verdeutlicht werden, wie sich der „Balanceakt des Alterns zwischen Fortschritt und Würde“ (Baltes 2007) in individuell differenter Weise vollzieht. Am Eintritt und Verlauf einer gravierenden sensorischen Kompetenzeinbuße (hier das Sehen) kann untersucht werden, welche Handlungsoptionen die Betroffenen institutionell und individuell-biografisch ausschöpfen, um mit dem Altern umzugehen. Die eingetretene chroni- 
sche Erkrankung wird in der Studie dabei als Ausgangspunkt für eine Fokusverschiebung betrachtet: Pläne für das Leben im Alter sind in der vorgestellten Art und Weise nicht mehr umsetzbar, da der Körper und seine Erkrankung in den Vordergrund tritt (Corbin u. Strauss 2004). Durch die Folgen des Sehverlusts wird eingeschränkte Handlungsfähigkeit (oftmals) zum ersten Mal im Alternsprozess spürbar: Zum Beispiel tritt die Realisierung von Ruhestandsprojekten in den Hintergrund und die Auseinandersetzung mit dem medizinischen System als Ort der Heilung, Behandlung und Therapie rückt ins Zentrum. Hinzu kommt in fortgeschrittenen Stadien der Erkrankung das Zurückgeworfensein auf den Alltag und dessen Bewältigung, da die gewohnten und routinierten Fertigkeiten des Alltags zum Teil außer Kraft gesetzt oder zumindest gefährdet sind. Auf einer zeitlichen Dimension verschwimmt in diesem Fall die Differenzierung von drittem und viertem Lebensalter, da die Erkrankung bereits jede fünfte Person über 65 und jede dritte über 75 Jahren (vgl. Holz et al. 2003) betrifft.

\subsection{Fragestellung}

Eine Annäherung an die empirische Realität des Lernens Älterer wurde in der Studie durch die Frage nach dem Umgang mit altersbedingten Einbußen auf institutioneller und individueller Ebene erforscht. Es wurde untersucht, wie sich der Balanceakt des Alterns individuell-biografisch und im Zugriff auf institutionelle Angebote vollzieht. Ziel war es dabei, die unterschiedlichen Modi des Umgangs mit der Erkrankung, die sich bei Betroffenen und (professionellen) Experten beobachten lassen, zu rekonstruieren. Es sollte analysiert werden, in welchem Verhältnis diese Modi unterschiedlicher Akteure zueinander stehen. Dabei wurde der Umgang mit der Erkrankung zunächst deskriptiv beschrieben und erst anschließend aus erziehungswissenschaftlicher Sicht mithilfe des Konzepts pädagogischer Formen (vgl. 2.3) nach pädagogischen und nicht-pädagogischen Formen differenziert.

\subsection{Stichprobe und methodisches Vorgehen}

Die untersuchte Stichprobe setzt sich aus 15 leitfadengestützten Interviews mit älteren Betroffenen der AMD und fünf institutionellen Vertretern vorhandener Angebotsformen für ältere Sehbehinderte zusammen. Um das derzeit verfügbare Angebotsspektrum für ältere Sehbehinderte möglichst umfassend abzudecken, wurden fünf Experten mit unterschiedlichen Interventionsangeboten für Sehbehinderte befragt. Es handelte sich dabei um eine Augenärztin mit psychotherapeutischem Beratungsangebot, um einen Trainer in Orientierung und Mobilität (O\&M) sowie in Lebenspraktischen Fertigkeiten (LPF), ${ }^{2}$ um einen spezifisch fortgebildeten Optiker, um eine professionelle Leiterin einer Beratungsgruppe und um eine Leiterin einer Selbsthilfegruppe.

Alle befragten Betroffenen haben an Interventionsangeboten teilgenommen. Sie waren zwischen 69 und 90 Jahren alt. Sie unterscheiden sich in Bezug auf die Sehbehinderung hinsichtlich der Betroffenheitsdauer und des verbleibenden Sehrestes. Für die Samplezusammenstellung wurde auf eine Streuung im Hinblick auf Alter, Krankheitsdauer, Geschlecht und Teilnahme an unterschiedlichen Angebotsformen geachtet. In der Analyse erwiesen sich jedoch nicht die objektiven Daten der Betroffenen als zentral. Viel- 
mehr wurde die interne Kontrastierung im Sinne eines „theoretical sampling“ (Strauss 1998) auf der Grundlage der Differenz der Teilnehmer im Hinblick auf Deutung und Handlung mit der Erkrankung vorgenommen. So ist das Sample nicht als repräsentatives Sample zu bewerten. Es unterliegt einer Samplingstrategie, die darauf bedacht ist, eine möglichst große Vielfalt von Formen des Umgangs mit einer Kompetenzeinbuße im Alter sichtbar zu machen.

Mit den Betroffenen wurden problembezogene Interviews (Witzel 2000) geführt, die nach dem Umgang mit der Sehbehinderung im Allgemeinen und mit den Angeboten im Speziellen fragten und zudem eine Aufforderung zur Biografieschilderung mit einschlossen. Mit den Experten hingegen wurden Experteninterviews nach Meuser und Nagel (2002) geführt, die den Fokus auf die Gestaltung der Angebote und den Umgang mit den Teilnehmern legten. Die Analyse der Daten folgte dem methodischen Konzept der Grounded Theory (Strauss 1998). In mehreren ineinander greifenden Schritten der Codierung und Kategorisierung, welche mittels Kontrastierungen und Dimensionalisierungen durchgeführt wurden, konnte die theoriegeneriende Schlüsselkategorie des „Netzwerks der sozialen Welt der Sehbehinderung" gewonnen werden. Die Rekonstruktion dieses Netzwerks verweist darauf, dass in diese soziale Welt eine Vielzahl von pädagogischen Formen eingebettet ist, auf die von Experten und Betroffenen jeweils unterschiedlich Bezug genommen wird. Aber ihre Relationierung und Verknüpfung innerhalb der sozialen Welt der Sehbehinderung zeigt ebenso auf, dass man den Umgang mit einer altersbedingten Kompetenzeinbuße nicht angemessen begreifen kann, wenn man lineare Vorstellungen pädagogischen Handelns im Sinne von Steigerungsentwicklungen zugrunde legt. Es hat sich vielmehr gezeigt, dass pädagogische Handlungsmuster in sozialen Formen institutionalisiert sind, die eng mit nicht-pädagogischen Handlungsformen verschränkt sind.

Zur Erläuterung des oben Genannten werden im Folgenden die beiden zentralen Konzepte der sozialen Welt (Strukturierungsprinzip) und der pädagogischen Formen (sensibilisierende Konzepte) näher beschrieben.

\subsection{Theoretische Konzepte: Soziale Welt und pädagogische Formen}

Das Konzept sozialer Welten (Clarke 2007) als methodologisches Konzept zur Beschreibung von Handlungen in interaktionalen Zusammenhängen bietet die Chance, die pluralen Umgangsformen entlang von Kategorien zu beschreiben und relational zu verknüpfen. Das Konzept entstammt einer symbolisch-interaktionistischen Tradition und wurde eingeführt, um Fragen nach sozialen Aggregationen, deren Zusammenhalt und deren Strukturierung zu verfolgen (vgl. Strübing 2005). Nach Strauss (1998) lässt sich eine soziale Welt dann formal als „cultural area“ (Strübing 2005) bestimmen, wenn es sich um eine nicht unbedingt große, weitestgehend in sich geschlossene Gemeinschaft handelt, die sich elementar mit einer Sache beschäftigt, über Örtlichkeiten und Organisationen verfügt, wo ihre Aktivitäten stattfinden, und die eine spezifische Technologie besitzt, um ihre Aktivitäten durchzuführen (vgl. Strauss 1998, S. 293). „Soziale Welten richten sich an zentralen Problembeständen des jeweils thematischen Interaktionsfeldes aus und entwickeln für ihre Bearbeitung definierbare Kernaktivitäten." (Schütze 2002, S. 60).

Die untersuchte soziale Welt setzt sich unter dem Aspekt der sie prägenden Gemeinschaftsformation aus einer eher beruflich orientierten und einer eher betroffenheits- 
orientierten Gemeinschaft zusammen, die durch die elementare Beschäftigung mit der Sehbehinderung als Kernaktivität aufeinander bezogen sind. Diese Aktivitäten können örtlich sowohl in lebensweltlichen wie auch in institutionellen Kontexten (Interventionsangebote) verortet werden. Derzeit befinden sich die Organisationen der sozialen Welt der Sehbehinderung in einem Stadium der Ausdifferenzierung, deren weitere Entwicklung von allen Akteuren mit beeinflusst wird. Die eingesetzte Technologie sichert den Fortbestand und die Entwicklung der sozialen Welt der Sehbehinderung. Was die Technologie generell kennzeichnet, sind wechselseitige Zuschreibungsprozesse von Kompetenz und Defizit. Ihre Analyse ist wesentliches Ziel der Darstellung (vgl. 3).

Um diese Prozesse in ihrem erziehungswissenschaftlichen Gehalt beurteilen zu können, wurden für die Analyse des Umgangs mit Sehbehinderung pädagogische Formen (Kade u. Seitter 1996, 2007a, 2007b) als sensibilisierende Konzepte an das Material angelegt. Der Diskurs zur Universalisierung und Entgrenzung des Pädagogischen (Lüders et al. 1995) hat den Blick für neue Ordnungsstrukturen geöffnet, mit denen sich ein erweiterter Gegenstandsbereich der Erziehungswissenschaft differenziert vermessen lässt. Die von J. Kade und Seitter (1996, 2007a, 2007b; vgl. auch Prange u. Strobel-Eisele 2006) empirisch rekonstruierten Formen des Pädagogischen beschreiben auf der Seite der Vermittlungspraktiken und auf der Seite der Aneignungspraktiken (zu dieser Differenz vgl. Kade 1997) Wissensformen (beispielsweise für die Vermittlung: Pädagogisches Wissen als Adressaten-, Vermittlungs-, Überprüfungswissen und Wissen über Wissen), die eine Differenz zwischen pädagogischer und nicht-pädagogischer Kommunikation beobachtbar machen. Als sensibilisierende Konzepte verhelfen sie damit zur Verortung des Umgangs von Experten und Betroffenen in eher pädagogischen oder primär nicht pädagogisch strukturierten sozialen Welten.

\section{Darstellung der Ergebnisse: Die Technologie der sozialen Welt als Pluralität von Defizit- und Kompetenzzuschreibungen}

Die Ergebnisdarstellung fokussiert die maßgebliche Kategorie der Technologie der sozialen Welt auf drei Ebenen: Zunächst wird an einem Fall die Zuschreibung von Kompetenzen mit der Aneignung von Hilfeangeboten in Verbindung gebracht. Dann wird fallübergreifend auf die Deutungskontexte der Betroffenen eingegangen, um zu erläutern, in welchen Kontexten sich ihre darauf bezogenen Handlungen verorten lassen. Schließlich wird in einem dritten Schritt die akteursübergreifende Sicht von Betroffenen und Experten auf die Technologie der sozialen Welt dargestellt, um damit die prägnantesten Unterschiede in Bezugnahme auf das institutionelle Gefüge zu kennzeichnen.

3.1 Der Zusammenhang von Deutungskontext und Aneignung von Hilfeangeboten. Ein prägnanter Fall

Zunächst soll die mit der Teilnahme an einem Interventionsangebot verbundene Herstellung von Defizit- und Kompetenzzuschreibungen anhand zweier Interviewauszüge eines prägnanten Falls in aller Kürze erläutert werden. Der Fall ist insofern prägnant, als der Zusammenhang von Deutungskontext und Aneignung von Hilfsangeboten hier besonders 
deutlich beobachtbar ist: In den Interviews mit den Betroffenen sind für die Kategorie der Deutungskontexte diejenigen Sequenzen von Interesse, an denen die Sehbehinderung zum ersten Mal thematisiert wird. Im vorgestellten Fall stellt dies die Diagnose der AMD-Erkrankung dar. Sie wird im weiteren Verlauf des Interviews eindrücklich mit der Bedeutung des Hilfsangebotes verknüpft.

mein augenarzt ist inzwischen dann verstorben, dann kam ne neue dran, die leider nicht sehr viel geredet hat. jedenfalls ich musste mir eh vor zwei jahren das auge operieren lassen mit der em $\mathrm{mi}=\mathrm{m}$ grauen star [...] und ich musste aber ins krankenhaus, ich das konnt man nicht ambulant machen. ich sollte drin bleiben, weil man nicht wusste was für komplikationen kommen. [...] eh ich hab seitdem ein wahnsinnig kompliziertes sehen. und bei der nachuntersuchung ham se gesagT: na ja und ihre makula is ja auch schon ganz schön weit. Ho. Ho. hab ich gesagt, ja was jetzt? Und da hab ich zu meiner augenärztin gesagt, warum sie mir das nicht gesagt hat. und da hat sie gesagT: wieso, sie haben doch sowieso alles was man nicht haben sollte. so ungefähr. also es ist jemand sie ist wohl sehr gut und so, aber reden kann sie nicht sehr gut mit einem und das ist ein ein ganz schlimmes problem $(3 / 24-3 / 50)$

In der Erzählung wird deutlich, dass die Betroffene von ihrer Erkrankung auf eine Weise erfuhr, die sie für nicht angemessen hält. Trotz großer Kompetenz in der Bewältigung anderer Augenerkrankungen wird sie von den Ärzten als inkompetent adressiert: Wichtige Fakten der eigenen Erkrankung werden ihr vorenthalten. Disqualifiziert werden nicht die Ärzte, die die aktuelle Operation durchführten, sondern die niedergelassene Augenärztin, die regelmäßige Kontrollen durchführt. Der regelmäßige Kontakt macht sie zur Vertrauensperson, deren Aufgabe insbesondere die Aufklärung und Information der Patienten ist. Diese zentrale Aufgabe wurde aus Sicht der Betroffenen nicht erfüllt. Obwohl die Diagnose als krisenhaftes Moment dargelegt wird, wird nicht über die Folge für das eigene Leben berichtet. Allein die Fremdzuschreibung eines Defizits und die mangelnde Kommunikation werden angeführt. In der weiteren Folge des Interviews wird dies auf die Teilnahme an der Beratungsgruppe übertragen und somit das Defizit relativiert. So sagt die interviewte Person auf die nachhakende Frage, inwiefern sich ihre Erwartungen an die Gruppe erfüllt hätten:

erstens mal weil frau j. und ehm selbst sehr interessiert ist, so neugierig ist, was gibt es. eh was tut sich in der weltgeschichte was augenkrankheiten anbelangt. [...] manch eh na ja eh inzwischen sind wieder $=n$ paar jahre vergangen und es hat sich schon einiges getan, aber frau j. ist inter, sehr interessiert uns das mitzuteilen. (I: ja) und sie forscht auch nach und allein das ist ja schon interessant, nich, ehm es gibt ja doch viel möglichkeiten, sich eh zu unterstützen, was essen zum beispiel anbelangt, nich. grüne sachen soll man viel essen bei augenkrankheiten. es gibt gewisse medikamente, wo man unterstützen kann. (.) ob sie einem dann helfen, ob sie einem was bringen, das muss man dann selber rausfinden, nich. aber es sind doch möglichkeiten sich da ( $\mathrm{I}: \mathrm{h}=\mathrm{hm})$ und ehm wenn sie nicht $\mathrm{zu}$ so=ner selbsthilfegruppe gehen, woher sollen sie das dann wissen? (15/50-16/22) 
Bemerkenswert ist in dieser Interviewsequenz, dass die Betroffene nun nicht bei den weiteren Teilnehmern der Gruppe, ${ }^{3}$ sondern bei der professionellen Leiterin ansetzt. Ihr wird der Status der Vermittlerin von Informationen verliehen; und zwar bestimmter Inhalte: Forschung, Heilung und Netzwerkwissen. Die Beschreibung und Charakterisierung und damit die Engführung der Rolle der Leiterin bildet den Gegenpol zu den Erfahrungen, die die Interviewte mit den Augenärzten gemacht hat. Die Leiterin unterscheidet das Interesse an der Mitteilung ihrer Nachforschungen von den Augenärzten, die nicht mit der Betroffenen reden. Die vermittelten Inhalte beziehen sich alle auf wissenschaftsbasiertes Wissen zur Eindämmung der Erkrankung (Nahrung, Medikamente). Es geht nicht um die Bewältigung des Alltags, sondern um die Vermittlung von Wissen und Hoffnung im weitesten Sinne. Die Betroffene eignet sich das ihr vermittelte Wissen in dem Sinne an, dass aktiv bleiben solle, auch weil es Fortschritte im Kampf gegen die Erkrankung gäbe (Medikamente, Möglichkeitsraum). Die Beratungsgruppe wird von dieser Betroffenen als Zwischeninstanz angesehen: Die Gruppe ist der Ort, an dem Wissen zur Ermöglichung von Handlungsoptionen zusammenläuft, auch wenn diese (noch) nicht wahrgenommen werden. Die Betroffene interpretiert die Eröffnung des Möglichkeitsraums als Hilfe zur Selbsthilfe. Es zählt nicht in erster Linie die Anwendung des angeeigneten Wissens zum Umgang mit der Sehbehinderung, sondern das Wissen über die sich eröffnenden Handlungsmöglichkeiten. Insgesamt repräsentieren diese Fallausschnitte einerseits die Umwandlung eines fremdzugeschriebenen Defizites in Kompetenz im Sinne des Zugriffs auf ein Angebot, andererseits stellt die Eröffnung von Handlungsmöglichkeiten weitere zu erwartende Defizite in einen relationierenden (abpuffernden) Zusammenhang. Erst durch die Zuschreibung spezifischer Defizite und Kompetenzen kommt es zur Nutzung eines Programms (institutionell) oder aber zu anderen Formen von Aneignung (individuell) zum kompetenteren Umgang.

\subsection{Deutungskontexte der Betroffenen-Typisierung}

Fallübergreifend ${ }^{4}$ lassen sich diese Zuschreibungen (Defizit vs. Kompetenz) an der Kategorie der Deutungskontexte (vgl. Abb. 1) verdichten. Diese bringt die wechselseitige Bezugnahme von Kontexten und Deutungen in den Blick, stellt damit die breite Einbettung der Erkrankung in den Lebenskontext dar und bildet die Grundlage für auf Defizitund Kompetenzzuschreibung beruhende Anschlussoptionen.

\subsubsection{Kontextualisierungen}

Die Kontextualisierungen betten die Deutungen auf medizinischer, biografischer, lebensalterbezogener und gesellschaftlicher Ebene ein:

(1) Wird die Erkrankung vor dem Hintergrund des Medizinischen thematisiert, sind die Aspekte Heilung, Behandlung, Therapie, (In-)Akzeptanz der Unheilbarkeit sowie die Kommunikation mit Ärzten von Bedeutung. Das Medizinische ist eng verknüpft mit der Deutung der Erkrankung und zumeist auch der Dauer der Erkrankung. Insbesondere diejenigen Betroffenen, die zum Zeitpunkt des Interviews erst kurz betroffen waren, fokussieren ihre Schilderungen auf medizinische Aspekte. Sie stellen die Sehbehinderung als Behandlungsgeschichte dar. Kürzlich betroffene Personen, für die das Medizinische eine 


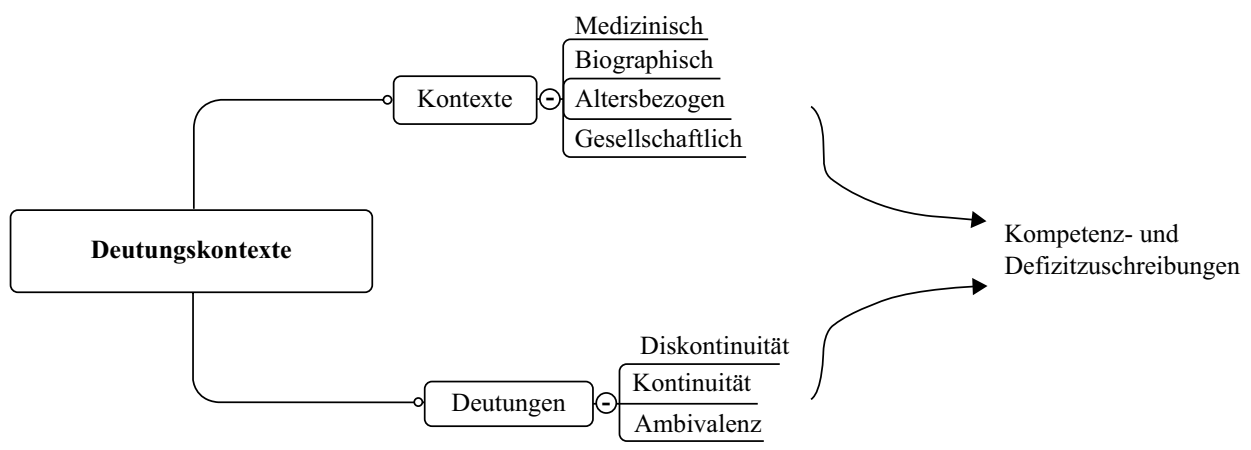

Abb. 1: Deutungskontexte der Betroffenen

untergeordnete Rolle spielt, erleben andere Erkrankungen (Parkinson, Nierenleiden) als gravierender und einschränkender im alltäglichen Leben. Bei Personen, die schon lange betroffen sind, ist mit Bezug auf das medizinische System vor allem die Enttäuschung omnipräsent.

(2) Sehr bedeutsam ist die Kontextualisierung der Erkrankung im Zusammenhang mit der Biografie. Auf der Ebene der Berufsbiografie stellt sich altersbedingte Makuladegeneration in einem Kontinuum vom Experten hin zum Leidenden dar. Das Erleben der Erkrankung wird hierbei der Berufsbiografie gleichgesetzt oder von ihr abgegrenzt, im Sinne einer Abwärts- bzw. Aufwärtskarriere. Während etwa im Fall eines Betroffenen die Erkrankung wie eine Managementaufgabe begriffen wird, und damit der Umgang mit der Sehbehinderung an die Berufsbiografie anschlussfähig ist, setzt sich eine Betroffene von ihrer Leidensbiografie des Berufslebens durch einen nun positiven Umgang mit der Sehbehinderung ab. Andererseits taucht die Sehbehinderung, mit Bezug auf die Berufsbiografie auch als Diskontinuität auf. Dies trifft insbesondere im Fall einer jüngeren Betroffenen (Jg.1945) zu. Bei ihr bricht die Sehbehinderung mitten ins Berufsleben ein und der progrediente Verlauf führt zur Aufgabe ihrer Lehrtätigkeit. Auf der Ebene von Krankheitsbiografien wird die Sehbehinderung relationiert mit früheren und momentanen Erkrankungen. Sie wird einerseits als Anschluss an überwundene Erkrankungen gedeutet, andererseits als Einbruch in eine ,Gesundheits'biografie. Was die Biografische Gesamtgestalt betrifft, kann der Zusammenhang zwischen Sehbehinderung und Biografie als Aufwärts- oder Abwärtstrend oder als zyklischer Verlauf beschrieben werden. Beispielhaft für einen zyklischen Verlauf ist folgender Fall einer Betroffenen: Die eigene Sehbehinderung wird mit der Kriegsblindheit des Ehemannes relationiert. Es bestehen keine Berührungsängste mit der sozialen Welt der Sehbehinderung, da die Blindheit des Mannes im Großen und Ganzen „familiär“ erlebt wurde. Durch die eigene Sehbehinderung kann an dieses Gefühl des Eingebettetseins angeschlossen werden.

(3) Eine weitere Dimension bildet die Auseinandersetzung mit dem Alter(n), dabei spielen die Themen Autonomie, Hilfsbedürftigkeit oder die Adressierung als alter Mensch eine wesentliche Rolle. Diesen Kontext repräsentiert etwa eine Betroffene, indem sie das Altern als Abbauprozess darstellt. Sie erlebt Verluste im Bereich Sehen und Hören als beeinträchtigend und kennzeichnet beide Beeinträchtigungen als Bestandteile des normalen Alternsprozesses. In einem anderen Fall hingegen wird das Eintreten der Sehbe- 
hinderung als unnatürlicher und verfrühter Alternsprozess erlebt: Altern scheint durch die Erkrankung als Grenze auf, die mit Verlust von Fähigkeiten, der möglichen Aufgabe der Selbständigkeit und einer Haltung generellen Loslassens verknüpft ist.

(4) Kennzeichnend für einen vierten Kontext ist die Thematisierung vor einem gesellschaftlichen Hintergrund. Es geht dabei im Besonderen um den neuen Status, den man als Behinderter in der Gesellschaft bekommt und die Angst vor sozialer Ausgrenzung. Besonders deutlich wird dies, wenn das Blindenzeichen thematisiert wird. Es wird als stigmatisierend erlebt, da die Betroffene nicht als Sehbehinderte , gekennzeichnet‘ werden will.

A: ich habe wohl dieses moderne abzeichen mit dem stock das internationale, aber ich geh nicht gern mit dem gelben abzeichen mit den drei punkten. Ich weiß nicht, es fällt den leuten noch mehr auf

$\mathrm{I}: \mathrm{h}=\mathrm{hm} \mathrm{h}=\mathrm{hm}$ also mit dem gehen sie eigentlich nicht aus dem haus

A: kaum. Ich hab da so=n ganz kleines. $(4 / 10-4 / 14)$

\subsubsection{Im Spannungsverhältnis von Kontinuität und Diskontinuität}

Die aus dem Interviewmaterial herausgearbeiteten Kontexte verorten die Deutungen der Erkrankung im Spannungsfeld von Kontinuität und Diskontinuität. Kontextualisierung und Deutung werden dabei jeweils individuell verknüpft.

Ein Beispiel der Herstellung von Kontinuität stellt eine Betroffene dar: Durch die Kriegsblindheit des Ehemannes ist ihr das Leben mit einer Sehbehinderung vertraut. Von größerer Relevanz ist die Erfahrung des Hörverlustes. Er wird als Erfahrung tiefgreifender biografischer Diskontinuität betont.

I: sind die augen die schlimmste einschränkung? oder sind andere dinge eher belastend?

B: äh, das gehör ist schlimmer.

I: das gehör ist schlimmer-

B: und das hat mein mann schon immer gesagt, [...] wenn ich nicht mehr hören könnte, das wäre viel schlimmer. ich kann mich ja noch unterhalten. mein mann war auch aufgeschlossen und- (.) ich kann mich mit jedem unterhalten und wenn mich halt einer übergeht, dem ist das auch passiert, [...] das- also das belastet mich mehr, wie das nicht sehen. (17/41-18/8)

Die Deutungsdimension der Diskontinuität kann als Destabilisierung bezogen sein auf Gegenwart, Vergangenheit oder Zukunft. Sie kann kurz-, mittel- oder langfristig ausgeprägt sein. Diskontinuitäten werden nicht nur durch die eigenmächtige Deutung der Personen ausgelöst, sondern teilweise durch die Meinungen Dritter weiter verschärft oder betont.

Die Herstellung von Kontinuität oder Diskontinuität ist aber im Verlauf einer Erkrankung nicht statisch. Vielmehr können diese Prozesse in der Zeit unterschiedlichen Bewertungen unterliegen. Bei einem Betroffenen steht die Diagnoseschilderung dafür, wie die stete Verschlechterung des Sehens zunehmend Aufmerksamkeit hervorruft. Biografisch ist 
relevant, dass der Übergang in den Ruhestand und der Ausbruch der Erkrankung zusammenfallen. Allerdings spürt er erst vier Jahre später massive Beeinträchtigungen. Das Eintreten der Unterscheidung krank/gesund und damit das Erleben von Diskontinuität werden erst durch diese Beeinträchtigungen und Erfahrungen von Hilflosigkeit relevant. ${ }^{5}$ Bezogen auf die Zuschreibung von Kompetenz ergibt sich somit in diesem Fall: Zunächst wird die Sehbehinderung als Defizit ignoriert, erst durch die tief greifende Erfahrung einer Grenze scheint ein Defizit auf. Dieses wird in diesem Fall mit dem biografischen Muster des ,Meisterns ‘ verknüpft, indem an das biografische Wissen um soziale Gruppierungen angeschlossen wird und er einer Beratungsgruppe beitritt.

Zusammenfassend zeigt die Analyse von Deutungskontexten, dass die Adressaten von Angeboten nicht als eine homogene Gruppe, sondern als eine in sich differenzierte soziale Realität gefasst werden müssen. Diese ist anschlussfähig an Kontexte, die durch sehbehindertenspezifische Interventionsangebote angesprochen werden, aber auch auf andere sehbehinderungsferne Kontexte bezogen. Damit verweisen die Deutungskontexte der Betroffenen auf eine erste Ausdifferenzierung sozialer Welten. Zur Erweiterung der Perspektive der Experten bedarf es noch weiterer Differenzierungen.

\subsection{Eingeschränkte Handlungsfähigkeit aus dem Blickwinkel von Experten und Betroffenen}

Die Relationierung der Perspektiven von Betroffenen und Experten zeigt die unterschiedlichen Referenzpunkte, die sich im Umgang mit eingeschränkter Handlungsfähigkeit aufdecken lassen (vgl. Abb. 2). In einem Netzwerk von Umgangsformen erfolgt die Erfüllung der Aufgabe der Sehbehindertenhilfe auf dem Weg der Wissensvermittlung, der Weitergabe von Informationen, der Präsentation von Hilfsmitteln, der Ermöglichung von Geselligkeit und der Weitervermittlung an andere Angebote. Soweit pädagogisches Wissen eingesetzt wird, kristallisiert es insbesondere um den Problemkomplex Veränderungsbereitschaft vs. Widerstände. Die unterschiedlichen Referenzpunkte auf der Ebene von Experten und Betroffen lassen sich anhand dreier Dimensionen herausarbeiten: der

Abb. 2: Schematische Übersicht

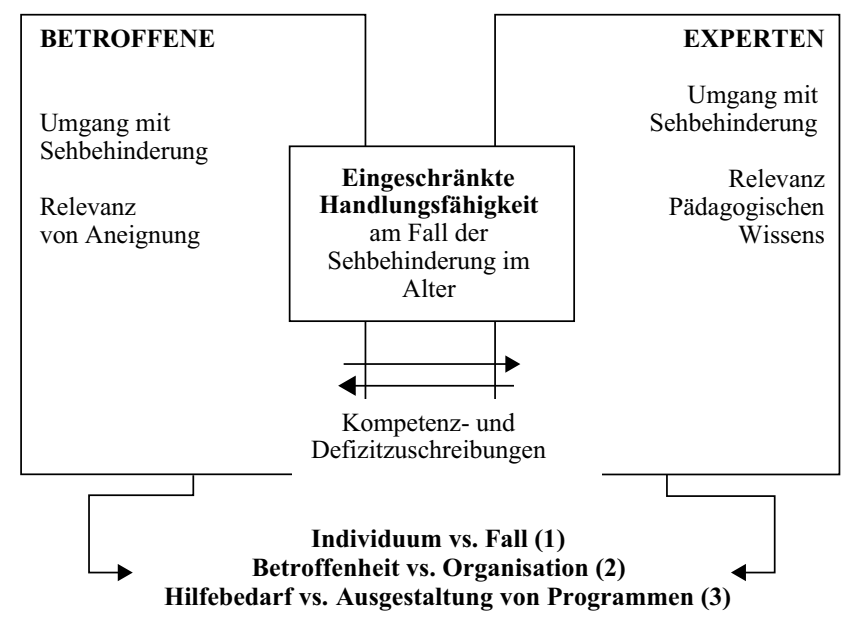


Ebene der Person (Individuum vs. Fall), dem Bezug auf das institutionelle Arrangement (Betroffenheit vs. Organisation) und dem Bezug auf die inhaltliche Ausgestaltung der Programme (Hilfebedarf vs. Ausgestaltung von Programmen). Die Aushandlungsprozesse auf diesen drei Ebenen prägen die soziale Welt der Sehbehinderung sowie deren Ausgestaltung.

(1) Die personale Dimension Individuum vs. Fall behandelt die Frage, wie sich die Akteure zur Sehbehinderung als Thema und damit zum Altern mit eingeschränkter Handlungsfähigkeit positionieren. Die Betroffenen beziehen sich aus ihrer je individuellen Lebenslage auf die Sehbehinderung (vgl. Deutungskontexte der Betroffenen). Es ist aber nicht die Deutung der Erkrankung allein, die die Betroffenen auf ein Angebot zugreifen lässt, vielmehr werden zunächst diese individuellen Kontexte bearbeitet und erst später wird die Aneignung eines Angebotes relevant. Das Spektrum des Erlebens der Erkrankung reicht dabei vom alleinig auf die Krankheit bezogenen Schicksalsschlag bis hin zur Kampfansage gegen das Altern im Allgemeinen. Diese unterschiedlichen Deutungen haben Handlungskonsequenzen. Das Krankheitserleben kann Handeln befördern oder behindern, zudem ist es nicht stabil über die Zeit und nicht unabhängig von der sozialen Einbettung. Aus den Interviews lassen sich folgende Phasen der Erkrankung idealtypisch rekonstruieren: Von einer Art Unglauben, über ein erstes Reagieren mittels Informations- und Therapiesuche nehmen die Betroffenen unterschiedliche Verläufe in Richtung Steigerung, Stabilisierung oder Erreichen einer Grenze.

Die Experten hingegen beziehen sich nicht auf diese individuellen Konstellationen (dies würde wahrscheinlich ihre Arbeit verhindern), sondern betrachten die Betroffenen als jeweils zu betreuenden Fall. Dies bedeutet, dass die Experten nur Teilbereiche der Problematik der Betroffenen in den Blick nehmen, und zwar diejenigen, die in Relation zum eigenen Programm stehen (optische Hilfsmittel, Alltagsgestaltung) oder den eigenen Interessen (Verkauf, Lobbyarbeit) entsprechen. Aus diesen unterschiedlichen Umgangsmodi mit einer Sehbehinderung ergeben sich zahlreiche Spannungen. Sie schlagen sich bei den Experten im jeweils selektierten Adressatenwissen nieder, in der Annahme von Unerreichbarkeit der Adressaten, der spezifischen Ausdifferenzierung von Methoden und in dem ihrem Programm jeweils dienlichen Zuschreibungen von Kompetenzen und Defiziten $^{6}$ an die Adressaten. In drei institutionellen Angeboten (Augenärztin, Training in lebenspraktischen Fertigkeiten [LPF], Beratungsgruppe) wird dennoch die Individualität der Betroffenen thematisiert: Im Fall der Augenärztin und des LPF-Trainings wird in vorgängigen Beratungsgesprächen geklärt, ob sich der Patient für weitere Beratungen oder ein Training eignet und welche individuellen Ziele verfolgt werden. Dabei wird auf das Krankheitserleben eingegangen, um die spätere Arbeitsgrundlage zu sichern. Die Thematisierung von Individualität hat hier selektiven Charakter. In der Beratungsgruppe werden individuelle Belange auf die Gruppe externalisiert, indem diejenigen Themen, die sich ausschließlich auf die Bearbeitung individuellen Umgangs beziehen, in den geselligen Austausch verlagert werden. Individualität wird hier einerseits der Rollenverteilung, andererseits zur Ermöglichung von Lehr-/Lernsituationen eingesetzt.

(2) Die institutionelle Dimension Betroffenheit vs. Organisation problematisiert die Unterschiede der Perspektiven hinsichtlich des Wunsches nach Bearbeitung der eigenen Betroffenheit, welcher der organisationellen Ausdifferenzierung der Experten entgegen- 
steht. Damit geht es um die Frage, wie der Umgang mit einer eingeschränkten Handlungsfähigkeit organisiert wird.

Die Experten thematisieren das Gesamtarrangement der Betreuung älterer Sehbehinderter, indem sie die Ausbildung institutioneller Netzwerke anstreben bzw. ablehnen. Es geht um die Gestaltung von Beziehungen oder Abgrenzungen zu anderen sozialen Welten, wie beispielsweise zum medizinischen System. Beziehungen zu anderen Professionellen werden aufgebaut, Trainingsmaßnahmen akkreditiert oder aber das eigene Angebotsprofil geschärft. Insgesamt geht es den Experten immer um den Fortbestand der eigenen Tätigkeit in Form der Legitimierung ihres eigenen Handelns und Professionalisierung. Der Bezug der Erkrankten auf die Organisation hingegen dient einzig dem Umgang mit der eigenen Betroffenheit im Sinne eines Anschlusses an die individuellen Deutungsmuster.

(3) In der inhaltlichen Dimension Hilfebedarf vs. Ausgestaltung von Programmen ist das pädagogische Wissen der Experten dem Umgang mit dem institutionellen Arrangement durch die Betroffenen gegenüberzustellen. Die Aneignung der Hilfsangebote erfolgt meist zufällig: Entweder wurden die Betroffenen von Bekannten oder Ärzten auf ein Angebot aufmerksam gemacht oder sie waren Teilnehmer einer (medizinischen) Informationsveranstaltung. Das Angebot trifft sie zumeist in ihrer Suchbewegung nach einer Therapiemöglichkeit und Hilfe. Dies läuft der Suchbewegung der Experten entgegen, die im Falle des Trainings oder des Optikers spezialisierte Angebote parat halten, oder zumindest Angebote, die nicht auf Heilung fokussieren, wie im Falle der analysierten Beratungsgruppe oder der interviewten Augenärztin. So müssen die Experten, bevor sie ihre Hilfsmaßnahmen beginnen, meist erst Aufklärungsarbeit leisten. In der Beratungsgruppe und bei der Augenärztin ist die Aufklärungsarbeit ein wesentlicher Bestandteil des Angebotes. Die unklaren Erwartungen der Teilnehmer stören dabei die Interaktion. Weniger dramatisch formuliert: Es müssen immer wieder Wege gefunden werden, die Voraussetzung für die Bereitschaft zur Annahme von Hilfsangeboten zu schaffen, und zwar durchwegs in einem konfliktreichen Aushandlungsprozess. Auf Expertenseite schlagen sich diese Störungen auf der Ebene des Adressatenwissens nieder: Am einen Ende des Extrems zeigt sich das Zielgruppenwissen durch Defizitzuschreibungen an die Adressaten. Es bezieht sich zumeist auf das Alter und damit einhergehende Einschränkungen: Ältere wollen bzw. können sich nicht mehr verändern, Ältere verstehen die Handhabung von Hilfsmitteln nicht, Ältere interessieren sich nicht für die inhaltliche Ausgestaltung von Angeboten, sondern ausschließlich für Geselligkeit. Oder aber die Defizitzuschreibungen beziehen sich auf die noch nicht geleistete Bewältigung der Erkrankung: Damit steht die Hoffnung auf Heilung der inhaltlichen Ausgestaltung des Programms im Wege. Am anderen Ende des Zielgruppenwissens stünden dann eher Formen, wie sie im Falle der Beratungsgruppe und des LPF-Trainings, in Form der Herstellung von Kommunikationsmöglichkeiten, zur Sprache kommen und die die inhaltliche Ausgestaltung der Programme beeinflussen.

Der Gesamtzusammenhang der pädagogischen Strukturierung der sozialen Welt der Sehbehinderung in der Relation Individuum/Institution verweist eher auf ein Kontinuum statt auf den eindeutigen Verweis von Defiziten und Kompetenzen. Defizit- und Kompetenzzuschreibungen verschieben sich über die Zeit, sind unterschiedlich eingebettet und produzieren dementsprechend unterschiedliche Bedarfe bei den Betroffenen. Die Betroffenen kennzeichnen sich durch plurale Aneignungsformen, die teilweise auf die 
pädagogischen Formen der Experten reagieren, teilweise diese ablehnen. Diese Aneignungsformen reichen von einem rein instrumentellen Umgang (Hilfsmittel, Hilfspersonen), über die Nutzung von Angeboten bis hin zu Geselligkeit. Die pädagogischen Formen der Experten im Sinne von Adressaten-, Vermittlungs- und auf die Aneignung bezogenes Vermittlungswissen changieren um die Problembereiche der Aushandlung von fallsensibler Bearbeitung der Veränderungsbereitschaft, aber auch der auftretenden Widerstände gegenüber krankheits- (und alters-)bedingt unumgänglichen Veränderungen.

Trotz dieser ersten Hinweise für eine umfassendere Betrachtung des Alterns in ihrem Verhältnis zu Bildung ist die Studie durch einige Limitierungen gekennzeichnet. Da alle Betroffenen an Interventionsangeboten teilgenommen haben, ist von einer positiven Selektion auszugehen. Denn eine Reihe von Personen haben diese gar nicht erst in Anspruch genommen. Zudem konnten nur die einzelnen Akteursperspektiven und ihre Relationierung dargestellt werden, die konkrete Untersuchung der Interaktion der Akteure in realen Vermittlungssituationen aber nicht vorgenommen werden. Dennoch geben die referierten Befunde wichtige Anregungen für eine (erziehungswissenschaftliche) Theorie über Lern- und Bildungsprozesse im Alter.

\section{Bildung Älterer in sozialen Welten}

Das Konzept der sozialen Welt liefert einen analytischen Zugang um einen erweiterten Bildungsbegriff für die erziehungswissenschaftliche Beschäftigung mit dem Altern empirisch vorzubereiten. Die referierte Studie hat nicht nur gezeigt, dass Aktivierung und Überwindung, sondern dass auch Prozesse des Hinausschiebens von Grenzen des Alterns in soziale Welten eingebunden sind, die sich als große Bandbreite von institutionellen Hilfeangeboten, sozialen Akteuren und medizinischem System entfalten. Aus der Sicht eines Bildungsbegriffs, der an Humboldt (1969) anknüpft, müsste damit Bildung (im Alter) in verschiedener Hinsicht erweitert werden: Zunächst sind Bildungsprozesse umfassender als Prozesse individueller Aneignung von (innerer und äußerer) Welt zu verstehen und damit von ihrer kognitiven Engführung auf Wissen zu befreien. Die Studie hat im Detail rekonstruiert, inwiefern die Aneignung von Welt nicht nur im Wissenserwerb besteht, sondern etwa auch die Integration in eine soziale Welt und in diesem Sinne die Aneignung der sich in ihr bietenden Lebensmöglichkeiten beinhaltet. Diesen Bildungshorizont kann man nur erschließen, wenn man die Aneignungssubjekte als konstitutiv in (unterschiedliche) soziale Welten eingebettete Individuen konzipiert und schließlich Bildungsprozesse mit den institutionellen Praktiken (von der Pädagogik bis zur Medizin reichend; sich an Defiziten oder auch Kompetenzen orientierend) relationiert, die mit ihren jeweiligen Technologien das „Leben“ in den jeweiligen sozialen Welten ermöglichen bzw. strukturieren. Ebenso handeln die Experten nicht als isolierte Individuen. Sie sind, wie die Betroffenen, in ihre speziell (professionell) sozialen, durch Vernetzung, aber auch Konkurrenz und Ausschließung geprägten Welten eingebettet. Vor diesem empirischen Hintergrund kann Altenbildung nicht nur als Bildung von Individuen mit ihren Möglichkeiten von Handlungserweiterung und Handlungsbegrenzung konzipiert werden. Sie manifestiert sich ebenso in der Gestaltung von Lebenswelten und institutionellen Settings, in denen Akteure und Betroffene agieren und aufeinander bezogen sind, um das 
Thema Altern in all seinen Facetten zu bearbeiten. Altern in einer spezifischen sozialen Welt ist somit strukturell, interaktionell und individuell, mehr oder weniger direkt oder diffus mit anderen sozialen Welten verkoppelt.

Altenbildung lässt sich aber nicht nur als Bildung begreifen, sie geschieht immer auch im Modus von Hilfe: In einer solchen Perspektive von Altenbildung spielen auch Settings eine Rolle, wie zum Beispiel das Hospiz, die vor allem auf das Hinnehmen von Abbauprozessen und somit Verlustregulationen setzen. Bislang sind diese beiden Modi der Altenbildung, Bildung und Hilfe, disziplinär eher getrennt: Während für Bildung die erziehungswissenschaftliche Sparte der Altenbildung zuständig zeichnet, wird Hilfe und der Umgang mit Krisen von der Sozialpädagogik bearbeitet (vgl. Kade 2001). Entsprechend ist der Bereich des Alterns bislang auch pädagogisch in drittes und viertes Lebensalter aufgeteilt. Am Fall der hier dargelegten Studie lässt sich aber aufzeigen, dass sich eine breite soziale Welt auf das Thema Altern entwickelt hat, die beide Bereiche umfasst. Dies legt die Vermutung nahe, dass sich derartige Übergänge und netzwerkartige Strukturen auch auf andere Bereiche des Alterns übertragen lassen und dies nicht einzig für den Bereich einer altersbedingten Sehbehinderung gilt. Diese Übergänge im Weiteren vor dem Hintergrund sozialer Welten zu untersuchen, kennzeichnet - so die hier vertretene These - das Programm einer weiteren Beschäftigung mit der sozialen Realität der Altenbildung. Ein Konzept von Altenbildung in sozialen Welten kann Impulse des lebenslangen Lernens aufnehmen, die das Individuelle wie das Institutionelle in ihrer Verschränkung und somit Relationalität zum Thema haben, denn die „Bildung des individuellen Subjekts in Form der Aneignung von Welt verläuft immer in der Verschränkung von individueller Lebensführung, Lehr-Lern-Arrangements, und (historisch spezifischen) gesellschaftlichen Verhältnissen (Kade et al. 2008, S. 11). ${ }^{7}$ So lässt sich der Gesamtkomplex des in der Studie zum Umgang mit der Sehbehinderung zum ersten Mal in seiner Differenziertheit zumindest ansatzweise aufgedeckte Umgang mit dem Prozess des Alterns in sozialen Welten als modernisierte Gestalt einer Altenbildung fassen, die sich in ein Konzept des Lebenslangen Lernens einfügt, das sich „lifelong“ und „lifewide“ (vgl. Alheit u. Dausien 2002) erstreckt.

Open Access Dieser Artikel unterliegt den Bedingungen der Creative Commons Attribution Noncommercial License. Dadurch sind die nichtkommerzielle Nutzung, Verteilung und Reproduktion erlaubt, sofern der/die Originalautor/en und die Quelle angegeben sind.

\section{Anmerkungen}

1 Mein besonderer Dank gilt an dieser Stelle den gutachtenden Personen dieses Artikels für die eingehende Kritik, die meine Überlegungen entscheidend weiter geklärt und teilweise auf ein neues theoretisches und methodisches Niveau gehoben hat.

2 Die Angebote O\&M und LPF werden in Deutschland von Rehabilitationslehrern für Sehbehinderte und Blinde durchgeführt. Ein Training in O\&M umfasst die Schulung am Blindenlangstock oder am weißen Stützstock und dient dem Erhalt der Mobilität. Dieses Training wird von den Krankenkassen übernommen. Ein LPF-Training muss in Deutschland privat getragen werden. Das Training dient der Aufrechterhaltung der selbständigen Lebensführung und umfasst die Schulung von Fertigkeiten im Umgang mit Alltagsgegenständen. 
3 Die Treffen der Gruppe sind inhaltlich zweigeteilt: Zunächst werden Informationen durch die Expertin vorgetragen, dann wird explizit auf den Austausch unter den Betroffenen gesetzt.

4 Der im Folgenden dargestellte fallübergreifende Überblick der Deutungskontexte der Betroffenen ist Resultat einer vergleichenden Beschreibung. Gewonnen wurde diese Beschreibung durch den Vergleich der aus den Einzelanalysen generierten Ergebnisse zu den Kategorien der Kontextualisierungen und der Deutungen.

5 Diese Diskrepanz erklärt sich, da erst ab einem Restsehvermögen von weniger als $30 \%$ auf dem besseren Auge Beeinträchtigungen gravierend hervortreten. Vorher kann das weniger betroffene Auge Defizite im Sehen gut ausgleichen.

6 „zu alt“, „zu depressiv“; ,,interessiert“; „Schaffer“/,Nicht-Schaffer“.

7 Zum Überblick über das lebenslange Lernen vgl. Hof 2009.

\section{Literatur}

Alheit, P., \& Dausien, B. (2002). Bildungsprozesse über die Lebensspanne und lebenslanges Lernen. In R. Tippelt (Hrsg.), Handbuch Bildungsforschung (S. 565-585). Opladen: Leske + Budrich.

Baltes, P. B. (1997). Die unvollendete Architektur der menschlichen Ontogenese: Implikationen für die Zukunft des vierten Lebensalters. Psychologische Rundschau, 48(4), 191-210.

Baltes, P. B. (2007). Alter(n) als Balanceakt: Im Schnittpunkt von Fortschritt und Würde. In P. Gruss (Hrsg.), Die Zukunft des Alterns (S. 15-34). München: Beck.

Baltes, P. B., Lindenberger, U., \& Staudinger, U. M. (2006). Life-span theory in developmental psychology. In R. M. Lerner (Ed.), Handbook of child psychology (Vol. 1: Theoretical models of human development, 6th ed., pp. 1029-1143). New York: Wiley.

BMFSJ (2005). Fünfter Bericht zur Lage der älteren Generation in der Bundesrepublik Deutschland. Berlin: Bundesministerium für Familie, Senioren, Frauen und Jugend.

Breinbauer, I. M. (2007). Bildung im Alter. In K. Aner, F. Karl, \& L. Rosenmayer (Hrsg.), Die neuen Alten - Retter des Sozialen? (S. 85-107). Wiesbaden: VS Verlag für Sozialwissenschaften.

Clarke, A. E. (2007). Social worlds. In G. Ritzer (Ed.), The Blackwell encyclopedia of sociology. (pp. 4546-4549). Malden, MA: Blackwell.

Corbin, J. M., \& Strauss, A. L. (2004). Weiterleben lernen. Verlauf und Bewältigung chronischer Krankheit. Bern: Huber.

Himmelsbach, I. (2009): Altern zwischen Kompetenz und Defizit. Der Umgang mit eingeschränkter Handlungsfähigkeit. Wiesbaden: VS Verlag für Sozialwissenschaften.

Hof, C. (2009). Lebenslanges Lernen. Stuttgart: Kohlhammer.

Holz, F. G., Pauleikoff, D., Spaide, R. F., \& Bird, A. C. (2003). Age-related macular degeneration. Heidelberg: Springer.

Humboldt, W. v. (1969). Theorie der Bildung. In W. v. Humboldt, Werke in fünf Bänden (Bd. 1). Darmstadt: Wiss. Buchgesellschaft.

Kade, J. (1997). Vermittelbar/nicht-vermittelbar: Vermitteln: Aneignen. Im Prozeß der Systembildung des Pädagogischen. In N. Luhmann \& D. Lenzen (Hrsg.), Bildung und Weiterbildung im Erziehungssystem. Lebenslauf und Humanontogenese als Medium und Form (S. 30-70). Frankfurt a.M.: Suhrkamp.

Kade, J., Hof, C., \& Peterhoff, D. (2008). Verzeitlichte Bildungsgestalten: Subjektbildung im Kontext lebenslangen Lernens. Report, 31(3), 9-22.

Kade, J., \& Seitter, W. (1996). Lebenslanges Lernen. Mögliche Bildungswelten. Erwachsenenbildung, Biographie und Alltag. Opladen: Leske + Budrich. 
Kade, J., \& Seitter, W. (2007a). Umgang mit Wissen. Recherchen zur Empirie des Pädagogischen. Bd.1: Pädagogische Kommunikation. Opladen: Barbara Budrich.

Kade, J., \& Seitter, W. (2007b). Umgang mit Wissen. Recherchen zur Empirie des Pädagogischen. Bd. 2: Pädagogisches Wissen. Opladen: Barbara Budrich.

Kade, S. (2001). Selbstorganisiertes Alter. Lernen in „,reflexiven Milieus “. Bielefeld: W. Bertelsmann.

Karl, U. (2008). Bildsamkeit und Bildungsprozesse im Alter. In K. Aner \& U. Karl (Hrsg.), Lebensalter und Soziale Arbeit: Ältere und alte Menschen (S. 161-173). Baltmannsweiler: Schneider Hohengehren.

Kolland, F. (2008). Lernbedürfnisse, Lernarrangements und Effekte des Lernens im Alter. In K. Aner \& U. Karl (Hrsg.), Lebensalter und Soziale Arbeit: Ältere und alte Menschen (S. 174-186). Baltmannsweiler: Schneider Hohengehren.

Lehr, U., Thomae, H., \& Diehl, M. (1987): Formen seelischen Alterns. Ergebnisse der Bonner gerontologischen Längsschnittstudie (BOLSA). Stuttgart: Enke.

Lüders, C., Kade, J., \& Hornstein, W. (1995). Entgrenzung des Pädagogischen. In H.-H. Krüger \& W. Helsper (Hrsg.), Einführung in die Grundbegriffe und Grundfragen der Erziehungswissenschaft (S. 207-215). Opladen: Leske + Budrich.

Mayer, K. U., \& Baltes, P. B. (1996). Die Berliner Altersstudie (Ein Projekt der Berlin-Brandenburgischen Akademie der Wissenschaften, Bd. 3). Berlin: Akademie-Verlag.

Meuser, M., \& Nagel, U. (2002). ExpertInneninterviews - vielfach erprobt, wenig bedacht: Ein Beitrag zur qualitativen Methodendiskussion. In A. Bogner, B. Littig, \& W. Menz (Hrsg.), Das Experteninterview - Theorie, Methode, Anwendung (S. 71-94). Opladen: Leske + Budrich.

Prange, K., \& Strobel-Eisele, G. (2006). Die Formen des pädagogischen Handelns. Stuttgart: Kohlhammer.

Schaie, K. W., \& Baltes, P. B. (1996). Intellectual development in adulthood. The Seattle longitudinal study. Cambridge: Cambridge Univ. Pr.

Schütze, F. (2002). Das Konzept der sozialen Welt im symbolischen Interaktionismus und die Wissensorganisation in modernen Komplexgesellschaften. In I. Keim \& W. Schütte (Hrsg.), Soziale Welten und kommunikative Stile (S. 57-83). Tübingen: Narr.

Staudinger, U. M. (2008). Was ist das Alter(n) der Persönlichkeit? Eine Antwort aus verhaltenswissenschaftlicher Sicht. In U. M. Staudinger \& H. Häfner (Hrsg.), Was ist Altern? Neue Antworten auf eine scheinbar einfache Frage (S. 83-94). Berlin: Springer.

Strauss, A. (1998). Grundlagen qualitativer Sozialforschung (2. Aufl.). München: Fink.

Strübing, J. (2005). Pragmatistische Wissenschafts- und Technikforschung. Theorie und Methode. Frankfurt a.M.: Campus.

Wahl, H., \& Heyl, V. (2004). Gerontologie - Einführung und Geschichte. Stuttgart: Kohlhammer.

Witzel, A. (2000). Das problemzentrierte Interview. Forum Qualitative Sozialforschung [Online Journal], 1(1), Art. 22. 